\title{
Les changements des horaires de fermeture des magasins et leurs conséquences sur les structures du commerce de détail
}

Shop closing-hour changes and their effects on retail trade structures

Veränderte Ladenschlußzeiten - veränderte Einzelhandelsstrukturen

\section{Günter Heinritz}

Traducteur : Birgit Brücher et Michel Deshaies

\section{(2) OpenEdition}

\section{Journals}

Édition électronique

URL : http://journals.openedition.org/rge/3992

DOI : $10.4000 /$ rge.3992

ISSN : 2108-6478

Éditeur

Association des géographes de l'Est

Édition imprimée

Date de publication : 1 janvier 2001

ISSN : 0035-3213

\section{Référence électronique}

Günter Heinritz, «Les changements des horaires de fermeture des magasins et leurs conséquences sur les structures du commerce de détail », Revue Géographique de l'Est [En ligne], vol. 41 / 1-2 | 2001, mis en ligne le 25 juillet 2013, consulté le 08 septembre 2020. URL : http://journals.openedition.org/ rge/3992 ; DOI : https://doi.org/10.4000/rge.3992

Ce document a été généré automatiquement le 8 septembre 2020

Tous droits réservés 


\title{
Les changements des horaires de fermeture des magasins et leurs conséquences sur les structures du commerce de détail
}

\author{
Shop closing-hour changes and their effects on retail trade structures \\ Veränderte Ladenschlußzeiten - veränderte Einzelhandelsstrukturen
}

Günter Heinritz

Traduction : Birgit Brücher et Michel Deshaies

\section{Introduction : les systèmes périodiques}

1 Tout se passe comme si la géographie du temps (Time- Geography) était à nouveau tombée dans l'oubli. Si la géographie humaine s'intéresse depuis toujours aux systèmes périodiques, surtout du point de vue spatial, en revanche, les recherches de géographie urbaine négligent injustement le facteur temps. Pourtant, les effets restrictifs de l'environnement temporel et spatial jouent plus que jamais un rôle important dans le cours de la vie des hommes. Ces facteurs qui influencent l'espace vécu, soit de manière cumulative, soit isolément, sont si importants que Pred (1977) évoque même la chorégraphie de l'existence. En tout cas, des changements dans le système d'activité de la population sont régulièrement causés par des changements du nombre, de la taille et de la distribution spatiale des «bundles " (lieux de vie ?) ou plutôt de leur accessibilité par le trafic ainsi que par les changements de leur accessibilité hebdomadaire et quotidienne. Si l'on se souvient de ces résultats de la recherche sur l'espace vécu, on s'étonnera que la géographie se soit si peu impliquée dans la discussion sur la libéralisation de la fermeture des magasins. Finalement, les lieux de vente du commerce de détail font sans aucun doute partie de ces «bundles» (lieux de vie ?) qui 
sont de très grande importance pour le comportement quotidien des consommateurs dans leur espace vécu.

\section{Le compromis du règlement sur la fermeture des magasins}

2 Dans le Reich de la fin du XIXe siècle (1891), la réglementation des horaires de fermeture des magasins reposait surtout sur des objectifs de politique sociale et de politique de concurrence. En revanche, la liberté d'action économique ou les besoins des consommateurs étaient secondaires. Cela est également valable pour ce qui concerne la loi sur les horaires de fermeture des magasins décidée en 1956 par le Bundestag, dont les structures fondamentales étaient restées en grande partie intactes pendant plus de 30 années. En conséquence, le marché et les consommateurs s'étaient largement accommodés du règlement législatif. C'est l'introduction de la soirée de service en 1989 qui a remis la question à l'ordre du jour. Contrairement à tous les autres règlements d'exception prévus par la loi, dont la validité ne s'applique qu'à des espaces (localités rurales, stations thermales, gares ou aéroports) ou à des périodes limitées (certains jours ouvrables et certains dimanches) ou qui ne concernent que certaines branches ou certaines formes d'activités (pharmacies, stations-service, kiosques), le jeudi soir était considéré en général comme soirée de service pour tous les lieux de vente dans l'ensemble de la République Fédérale. L'expérimentation de la soirée de service dont l'introduction avait été justifiée essentiellement par la demande croissante de dérogations à la loi de fermeture des magasins, par exemple pour les stations-service ou les gares dans les villes de plus de 200000 habitants, a joué naturellement un rôle important dans la discussion de la nouvelle loi, rôle sur lequel nous allons maintenant revenir.

\section{La loi sur les horaires de fermeture des magasins à l'essai : le rapport IFO (1995)}

3 Au fil du débat sur la déréglementation, la brèche ouverte par l'introduction de la soirée de service a offert un bon point de départ pour remettre en question la justification politique de la réglementation des horaires de fermeture des magasins. Le Ministère Fédéral du Travail et de l'Ordre Social et le Ministère Fédéral de l'Économie ont chargé d'une étude l'Institut IFO qui a présenté en août 1995 ses « réflexions sur la politique du commerce intérieur et de la concurrence ainsi que sur la politique de l'emploi et la réglementation du travail » sous le titre « La loi allemande sur les heures de fermeture des magasins à l'épreuve ».

Dans ce rapport sont rassemblés non seulement les arguments qui sont donnés dans la discussion politique et dans les ouvrages d'économie ainsi que les expériences tentées avec les règlements d'exception à la loi sur les heures de fermeture des magasins, mais aussi les résultats de certaines enquêtes menées par l'institut IFO. Dans l'ensemble de la République Fédérale entière, l'Institut IFO a sélectionné et questionné par écrit des entreprises individuelles, des consommateurs ainsi que des salariés du commerce de détail et d'autres commerces afin d'obtenir une base de données actuelle et représentative pour leurs propres recommandations. 
5 Bien que tous les sondages aient dégagé une forte majorité pour la conservation de la loi en vigueur alors sur les heures de fermeture des magasins, les chercheurs de l'Institut IFO ont constaté un intérêt croissant pour une flexibilité accrue des horaires d'ouverture des magasins, surtout chez les consommateurs qui sont pris par leur travail ou par leurs enfants ainsi que chez les salariés pour qui les soirées de travail peuvent mieux s'harmoniser à leur situation personnelle. Des propriétaires de grandes surfaces avec un chiffre d'affaires important ainsi que de jeunes commerçants indépendants favorables aux innovations se sont également déclarés intéressés. Malgré tout, plus de la moitié des entreprises indépendantes questionnées se sont déclarées contre la libéralisation des horaires d'ouverture des magasins. Pourtant, la part des partisans (40\%) était plus élevée que lors des sondages précédents. Les jugements sur la libéralisation des horaires d'ouverture par le commerce de détail dépendaient des évolutions de la concurrence ainsi que des possibilités des entreprises d'aménager les horaires d'ouverture des magasins. L'importance du chiffre d'affaires, la branche d'activité et la forme de l'entreprise, ainsi que le lieu d'implantation, se sont révélés comme étant des facteurs importants pour les ajustements prévus. Parmi les arguments récurrents invoqués par des entrepreneurs indépendants contre une libéralisation des horaires d'ouverture des magasins, l'Institut IFO a recueilli surtout les frais de personnel et d'entreprise supplémentaires, l'insuffisante affluence des clients pendant les heures de la soirée. Les arguments les plus importants des partisans étaient la concurrence, c'est-à-dire la possibilité de s'adresser à de nouveaux clients le soir grâce à l'ouverture prolongée des magasins. Ce sont surtout des entreprises à chiffre d'affaires important implantées en périphérie qui ont évoqué cette possibilité comme celle d'une forte affluence de clients attendue le soir. En revanche, les propriétaires de petits magasins ou de magasins de taille moyenne dans les centres ville ne comptent sur aucun effet positif de la prolongation des heures d'ouverture le soir. En plus, les questions posées aux commerçants font comprendre que les heures d'ouverture des magasins qui, jusque là, ont été quasiment sans influence dans la concurrence, joueraient un rôle important dans le cas d'une libéralisation, surtout entre les grandes surfaces.

6 Les questions posées aux consommateurs ne font pas non plus ressortir de majorité pour un changement de la loi de fermeture des magasins : au total, seulement un tiers à un cinquième de tous les consommateurs interrogés se sont déclarés pour un prolongement de l'ouverture des magasins le soir ou bien le samedi après-midi. Les quatre-cinquièmes de l'ensemble des personnes interrogées ont déclaré pouvoir réaliser leurs courses sans stress et sans problème pendant les horaires d'ouverture existants. Seulement $20 \%$ ont déclaré avoir rencontré des difficultés à faire leurs courses en raison de la faible durée d'ouverture des magasins et qu'on pourrait les aider avec une libéralisation des heures de fermeture.

7 Comme dans cette hypothèse les heures de travail étaient également prolongées pour les employés du commerce de détail, un troisième sondage de l'Institut IFO s'est adressé à des salariés travaillant ou non dans le commerce de détail. Ce sondage a révélé que la plupart des employés du commerce de détail considèrent le prolongement des heures d'ouverture comme un préjudice à leurs conditions de travail. Pourtant, il est apparu aussi qu'il existait un potentiel important d'employés susceptibles de travailler plus longtemps le soir ainsi que le samedi. 
8 En conclusion de son étude l'Institut IFO émet une recommandation nettement en faveur d'une libéralisation des heures d'ouverture. Pourtant, les résultats des sondages donnent l'impression qu'un changement de la loi sur les horaires de fermeture des magasins n'était pas utile. C'est donc par une décision politique que l'on a dû fixer le cadre juridique déterminant les heures où l'on peut faire ses achats. C'est-à-dire qu'il fallait décider si on devait simplement tenir compte des majorités absolues ou bien des minorités qui étaient intéressées par la prolongation des heures d'ouverture. L'institut IFO a justifié sa recommandation de la façon suivante : «L'élargissement de la liberté d'action des entreprises, des consommateurs ainsi que des salariés promet des améliorations importantes et remarquables, surtout pour les acteurs qui sont actuellement défavorisés par la loi de fermeture des magasins. Cela est conforme à l'esprit d'un ordre économique orienté vers la compétition que de laisser plus d'espace aux forces d'action favorables à l'innovation, plutôt que de conserver des structures héritées des règlementations des quatre dernières décennies limitant la liberté d'action. (U. Chr. Täger, K. Vogler-Ludwig u. S. Munz 1995).

\section{Les expériences de la soirée de service}

9 Finalement, les auteurs de cette étude attendaient des effets positifs d'une libéralisation de la fermeture des magasins sur le plan économique global. Certes, les auteurs considèrent comme limité le potentiel d'une expansion significative de la consommation privée concernant le commerce de détail. Mais pourtant, ils pensent que le chiffre d'affaires du commerce de détail devait augmenter de 2 à $3 \%$ en 3 ans grâce à la libéralisation des heures d'ouverture. Ils évaluent les effets d'emploi qui y sont liés à $1,3 \%$ des employés du commerce de détail, c'est-à-dire à environ 50000 personnes ou 31000 équivalents de salariés à temps complet. En l'occurrence, ils pouvaient se baser sur des expériences positives correspondantes avec la libéralisation des heures d'ouverture en Europe, par exemple en Suède. Là, les effets sur l'emploi ainsi que les effets sur le chiffre d'affaires observés étaient à peu près du même ordre.

Bien évidemment, ces effets de nature économique globale ne représentent pas un avantage équivalent pour chaque entreprise commerciale. Cependant, ils sont différenciés de manière nette selon les types d'entreprises ainsi que selon leurs lieux d'implantation. Quant aux types d'entreprises, l'étude révèle que la libéralisation des heures d'ouverture profite surtout aux grandes surfaces, c'est-à-dire les hypermarchés ainsi que les grandes surfaces spécialisées. En revanche, les commerces spécialisés de taille moyenne perdent du chiffre d'affaires. Une enquête sur les conséquences de la soirée de service donne également des résultats très différents (A. Wölk 1993) selon le type d'entreprise, la branche concernée ainsi que sa localisation. C'est parce que le succès de la soirée de service auprès des consommateurs est soumise à des conditions dont ne bénéficient pas toutes les implantations. Afin d'être attractive pour les consommateurs lors de la soirée de service, une implantation doit :

1. pouvoir présenter une offre attirante et diverse. C'est pour cela que l'on préfère les localisations où se trouvent déjà des entreprises de forte attraction (des grands magasins, également de grands self-service et des magasins spécialisés) dont les offres de commerces de détail sont complétées par des offres de services (gastronomiques et culturelles);

2. présenter une participation à la soirée de service de toutes les entreprises ainsi que des heures d'ouverture homogènes ; 
3. être facilement accessible, c'est-à-dire offrir des parkings et de bonnes correspondances de bus.

11 Ces conditions sont surtout celles des lieux de stationnement très centraux ou alors (quant à l'accessibilité par voiture) des surfaces de vente « auf der grünen Wiese ${ }^{1}$.

De plus, les lieux principaux à soirée de service étaient situés dans les grandes villes et dans les centres moyens de l'espace rural (à condition que ces derniers soient plus facilement accessibles que le centre supérieur le plus proche et qu'ils présentent une offre de commerce attrayante). Dans ce cas, la participation à la soirée de service se concentrait surtout sur les meilleures localisations du centre ville. La participation à la soirée de service dans les localisations marginales du centre ville était faible.

13 Pour les grandes surfaces, un deuxième pôle de fréquentation se trouvait «auf der grünen Wiese ». L'avantage des centres commerciaux repose sur la concentration de magasins de branches très variées et sur la concordance des heures de fermeture. Tandis que les clients qui visitaient de telles implantations pendant la soirée de service étaient venus avec la ferme intention d'acheter, dans les centres ville au contraire on venait non seulement pour les achats, mais également dans une large mesure avec l'intention de flâner et de regarder les vitrines. En 1992, cinq ans après l'introduction de la soirée de service, environ $35 \%$ des entreprises y participaient, et on avait donc ainsi enregistré un recul d'environ $15 \%$ par rapport au début. On a remarqué une interaction entre le degré de participation des entreprises à la soirée de service et le succès rencontré par cette dernière auprès des clients: moins les entreprises participent, moins l'offre est diverse et par conséquent moins les magasins attirent les clients. D'où une diminution de la participation des commerces de détail. En ce qui concerne les conséquences de la soirée de service sur le plan spatial, A. Wölk (1993) montre :

1. qu'elle a conduit à une animation des centres ville et à une augmentation de l'attractivité de ces villes,

2. qu'elle s'est effectuée sans aucun doute en faveur des lieux centraux supérieurs,

3. qu'elle a eu pour conséquence une augmentation du trafic individuel le jeudi soir dans les centres-villes, notamment là où les transports publics ne se sont pas adaptés.

\section{Les premières expériences avec les nouvelles heures d'ouverture}

14 Certes, on ne peut pas simplement transposer les expériences de la soirée de service et d'autres exceptions à l'ancienne loi sur les heures de fermeture des magasins aux effets de la prolongation générale des heures d'ouverture (Tableau 1). Mais il ne faisait pas de doute que l'élargissement du cadre temporel ne serait pas automatiquement utilisé partout et par tout le monde. Ceci s'est tout de suite confirmé après l'entrée en vigueur de la nouvelle loi qui permet une ouverture le soir jusqu'à $20 \mathrm{~h}$ (la loi étant nettement en retrait par rapport à la recommandation du rapport IFO qui avait proposé une ouverture quotidienne jusqu'à $22 \mathrm{~h}$ ). En effet, peu d'entreprises ont saisi la possibilité de laisser le magasin ouvert jusqu'à 20h. Les consommateurs n'ont pas non plus profité entièrement des nouvelles possibilités : Selon les observations du Service de Presse du GfK du 06-03-1997, en novembre et décembre 1996, 55 \% des consommateurs n'avaient pas du tout profité des nouvelles heures d'ouverture des magasins, $15 \%$ en avaient 
profité une fois, $30 \%$ deux fois ou plus. À la vérité, des heures d'ouverture non homogènes ont souvent mené à la confusion des consommateurs. Déjà en décembre 1996, les journaux parlent de «farces provinciales dans les heures de fermeture des magasins » et de « chaos total ». Les semaines suivantes, ils annoncent que la nouvelle réglementation s'effiloche déjà et que le commerce de détail commence à se désengager.

Tableau 1 : Comparaison entre les anciennes et nouvelles heures de clôture des magasins

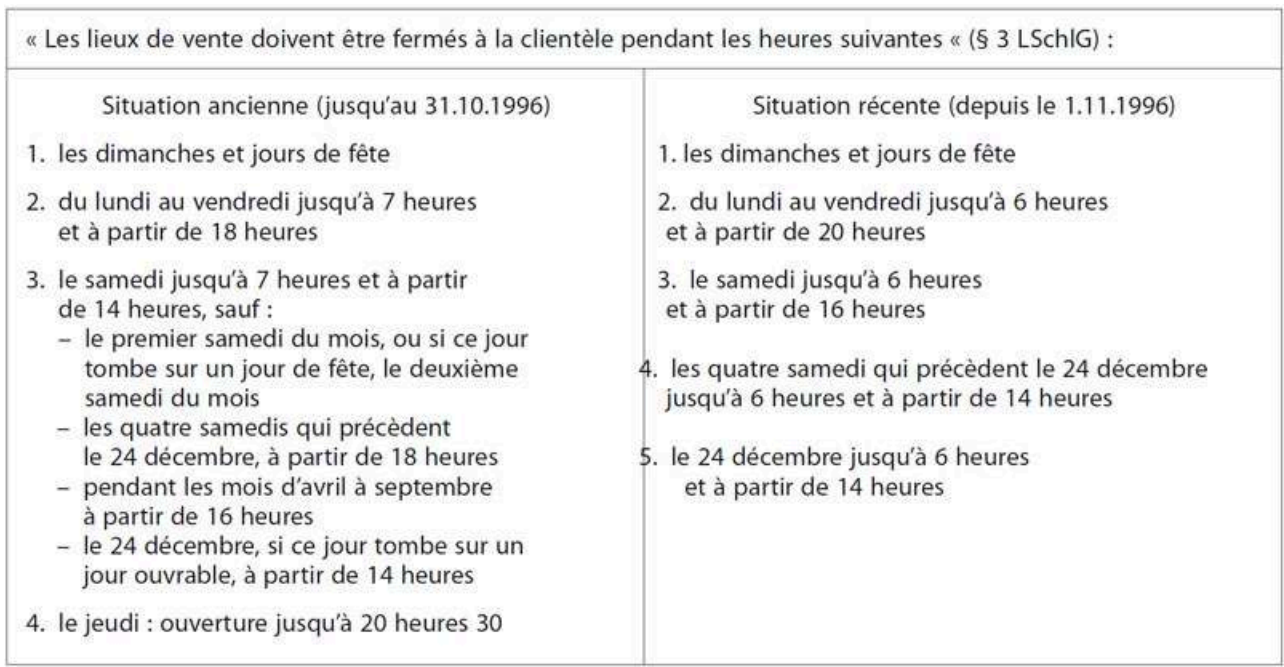

Source : Extrait de la loi de la fermeture des magasins du 28 novembre 1956, dans les formules du 25 juillet 1986 et du 19 juillet 1996. [Tiré des études IFO 58]

Peu de temps après l'entrée en vigueur des nouveaux règlements, des rapports concluant au succès ou à l'échec ont été lancés par les représentants des différents partenaires Le 25 février 1997 par exemple, les services de presse du syndicat HBV à Munich émettent le doute, sous le titre « 100 jours de la nouvelle loi sur l'ouverture des magasins », que le commerce de détail munichois puisse maintenir sa devise ("C'est promis : plus jamais de fermeture avant 20 heures ») et constatent qu'une réduction des heures d'ouverture le soir s'impose d'urgence. Inversement, lors de son congrès les 24/25 février à Cologne, l'union fédérale des exploitants de succursales et des magasins self-service s'oppose à « la tentative de mettre en doute les effets positifs sur le chiffre d'affaires et les employés ». L'union fédérale trouve que les premières réactions des clients sont tout à fait encourageantes « de sorte qu'il serait souhaitable de prolonger les phases de test pendant un an ».

Ainsi, peu de mois après l'application des nouveaux règlements, on ne pouvait décider définitivement lequel des arguments avancés dans les discussions précédentes s'était révélé concluant. Mais avec l'adoption de la nouvelle loi en 1996, le législateur avait ordonné au gouvernement de fournir un rapport, dans lequel les expériences sur les effets des règlements de la fermeture des magasins devaient être présentés trois ans après l'entrée en vigueur de la loi. Ce rapport qui a été élaboré par l'Institut IFO à Munich se base notamment sur les sondages effectués auprès des consommateurs et des entreprises réalisés au printemps 1999. Il en résulte que le degré d'acceptation du règlement actuel par les consommateurs a progressé légèrement, jusqu'à $57 \%$. Plus les interrogés sont jeunes, plus leur niveau de formation est élevé et plus leur commune de résidence est peuplée, plus le degré d'acceptation est élevé. Comme on pouvait s'y 
attendre, l'acceptation du nouveau règlement est au-dessus de la moyenne chez les consommateurs qui sont pris par leur profession ou bien par leurs enfants.

Cependant, l'acceptation ne prouve pas pour autant que les consommateurs participent et qu'ils adaptent leur comportement d'acheteur. En effet, les résultats des sondages effectués auprès des consommateurs montrent que seulement $42 \%$ des interrogés profitent des trois premiers jours de la semaine pour faire leurs achats (fig. 1).

Figure 1 : Utilisation des nouveaux horaires d'ouverture des magasins par les consommateurs indication des consommateurs interrogés en \%
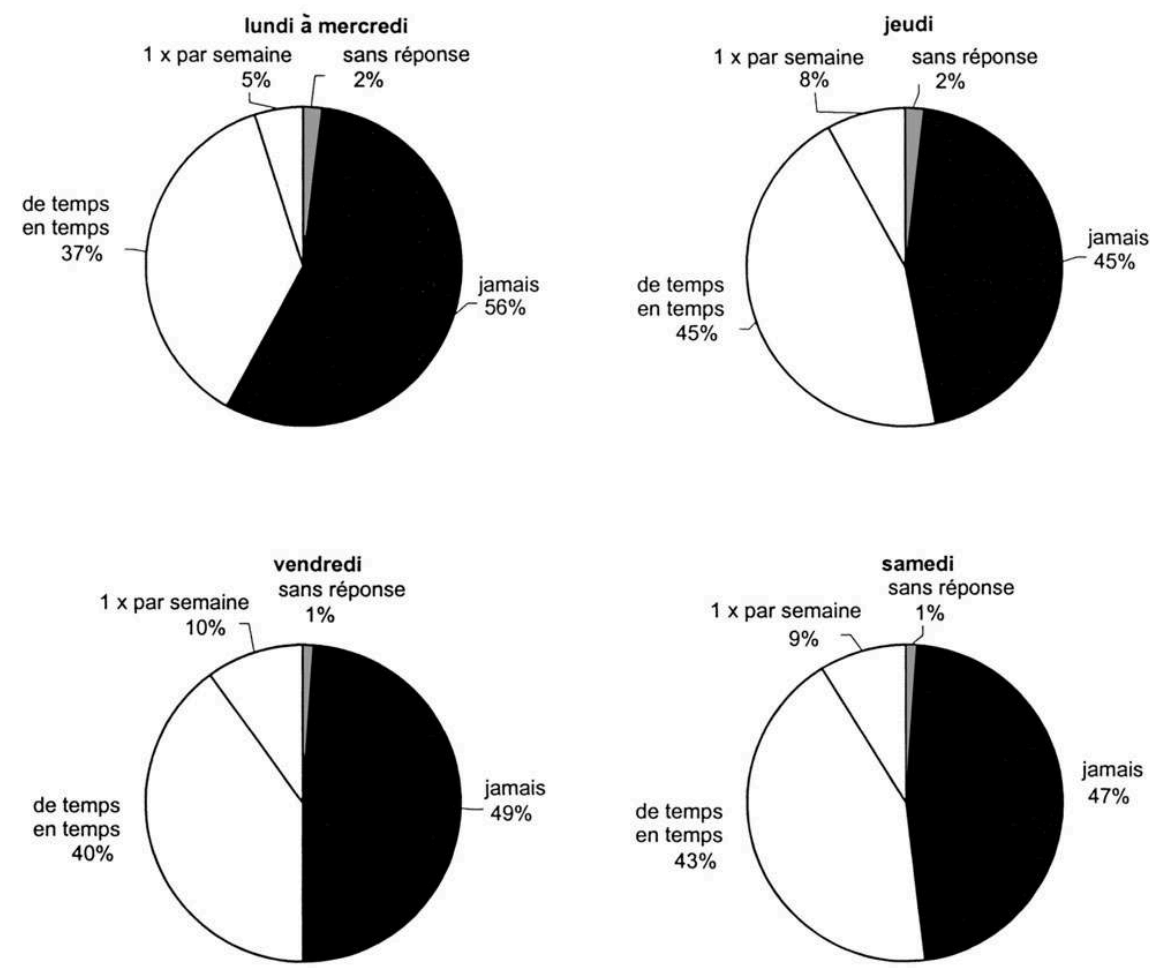

Source : ifo/test infra - sondage des clients à propos des horaires de fermeture des magasins 1999.

Entre 49 et 53 \% des personnes interrogées font effectivement leurs achats le jeudi et le vendredi soir, mais surtout le samedi après-midi. Ces taux sont nettement plus élevés (entre 54 et $57 \%$ ) dans les villes de plus de 500000 habitants parce que les types de commerces qui se montrent prêts à ouvrir plus longtemps sont plus nombreux et que les consommateurs préfèrent de plus en plus les ensembles de magasins situés en ville, qui se prêtent spécialement à combiner achats et distraction du soir. Durant les heures prolongées d'ouverture, il y a un rapport étroit entre la localisation, la taille du commerce et le type de produit qu'il propose. D'un côté, le samedi après-midi, les consommateurs préfèrent nettement acheter les vivres au supermarché s'il y en a un dans leur quartier. Par contre, le soir, ils préfèrent les magasins en position bien centrale dans une agglomération animée, où ces derniers ont nettement renforcé leur activité de publicité et où effectivement $90 \%$ des entreprises pratiquent les heures d'ouverture prolongées. Vu globalement, toutes les entreprises ne le font pas. La moyenne d'heures d'ouverture par semaine, qui était de 45 heures en Allemagne en 1995, n'a augmenté que de 5 heures au total. Les questions posées aux entreprises par l'Institut IFO révèlent qu'environ un quart seulement de toutes les entreprises 
pratiquent vraiment les heures d'ouverture prolongées permises par la loi au moins deux jours de la semaine et le samedi après-midi. Ces magasins représentent environ $62 \%$ du chiffre d'affaires du commerce de détail en Allemagne (Tableau 2).

Tableau 2 : Intensité d'utilisation des heures d'ouverture des magasins prolongées par la loi dans le commerce de détail, selon la classe de la taille du chiffre d'affaires

\begin{tabular}{|c|c|c|c|c|c|}
\hline \multirow{3}{*}{$\begin{array}{l}\text { Classe de taille } \\
\text { du chiffre d'affaires }\end{array}$} & \multicolumn{4}{|c|}{$\begin{array}{l}\text {... \% des lieux de vente } \\
\text { profitant du prolongement des heure } \\
\text { d'ouverture le soir et le samedi }\end{array}$} & \multirow{3}{*}{$\begin{array}{l}\text { Part des lieux de } \\
\text { vente dans } \\
\text { la classe de la } \\
\text { taille du chiffre } \\
\text { d'affaires }\end{array}$} \\
\hline & \multicolumn{3}{|c|}{$\begin{array}{l}\text { du lundi au vendredi } \\
\text { (après } 18 \mathrm{~h} \text { ) }\end{array}$} & \multirow[t]{2}{*}{$\begin{array}{l}\text { Le samedi } \\
\text { (après } 14 \mathrm{~h} \text { ) }\end{array}$} & \\
\hline & $\begin{array}{l}\text { au moins } \\
\text { deux fois }\end{array}$ & $\begin{array}{l}\text { au moins } \\
\text { trois fois }\end{array}$ & $\begin{array}{l}\text { au moins } \\
\text { quatre fois }\end{array}$ & & \\
\hline moins de 250000 & 27,2 & 25,2 & 23,7 & 23,0 & 30,1 \\
\hline $250000 \leq 1$ million & 34,3 & 29,0 & 25,4 & 25,0 & 40,7 \\
\hline 1 million $-\leq 2$ millions & 43,9 & 33,3 & 27,4 & 31,1 & 13,6 \\
\hline 2 millions $-\leq 5$ millions & 55,6 & 45,9 & 41,0 & 36,8 & 9,9 \\
\hline 5 millions $-\leq 25$ millions & 87,1 & 81,1 & 76,0 & 77,4 & 5,1 \\
\hline 25 millions $-\leq 50$ millions & 97,5 & 92,5 & 86,2 & 95,6 & 0,3 \\
\hline 50 millions $-\leq 100$ millions & 99,1 & 98,3 & 96,5 & 98,3 & 0,2 \\
\hline 100 millions et plus & 98,7 & 98,7 & 98,7 & 98,7 & 0,1 \\
\hline Total & 38,6 & 33,2 & 29,7 & 28,8 & 100 \\
\hline
\end{tabular}

Source : ifo/sondage sfs dans le commerce de détail (1999), concept d'entreprise.

En raison des conditions défavorables de vente et des frais, ce sont surtout les entreprises les plus petites (avec un chiffre d'affaires inférieur à 2 millions de DM par an), gérées directement par leurs propriétaires, qui n'ont pas de profit à attendre de la prolongation des heures d'ouverture. Leur horaire moyen d'ouverture est de 48 heures, tandis que les entreprises qui ouvrent plus longtemps ont augmenté leur horaire jusqu'à 58 heures. Celles-ci se trouvent surtout dans des emplacements très passants, notamment dans des centres commerciaux, où les heures d'ouverture sont prévues par le contrat de location (Tableau 3).

Tableau 3 : Utilisation des heures d'ouverture prolongées par des magasins selon les localisations

\begin{tabular}{|c|c|c|c|}
\hline \multirow[b]{2}{*}{ Localisation } & \multicolumn{3}{|c|}{$\ldots \%$ des magasins profitant des heures d'ouverture prolongées } \\
\hline & $\begin{array}{c}\text {... au moins } 2 \text { fois, } \\
\text { du lundi au vendredi } \\
\text { après } 18 \mathrm{~h} 30 \text { et le samedi }\end{array}$ & $\begin{array}{c}\text { Au moins } 2 \text { fois } \\
\text { du lundi au vendredi } \\
\text { après } 18 \mathrm{~h} 30\end{array}$ & Le samedi après $14 \mathrm{~h}$ \\
\hline Centre ville/ city & 29,3 & 37,0 & 39,7 \\
\hline Banlieue, faubourg & 18,2 & 32,3 & 20,7 \\
\hline $\begin{array}{l}\text { Zone d'activité en dehors } \\
\text { de l'agglomération }\end{array}$ & 52,2 & 56,7 & 53,8 \\
\hline Petite commune rurale & 10,6 & 20,7 & 12,8 \\
\hline Centre commercial & 65,9 & 74,9 & 70,4 \\
\hline $\begin{array}{l}\text { Voisinage avec d'autres } \\
\text { magasins }\end{array}$ & 18,5 & 29,5 & 24,0 \\
\hline Zone piétonne & 35,1 & 42,2 & 49,7 \\
\hline To tal & 23,2 & 33,2 & 28,8 \\
\hline
\end{tabular}

Source : ifo/sfs : sondage du commerce de détail (1999), concept d'entreprise 

jouent un rôle important que pour une petite minorité d'entreprises quant au règlement d'ouverture des magasins. La stratégie d'ouverture d'un magasin s'oriente dans une large mesure en fonction des concurrents, surtout si ceux-ci utilisent les ouvertures le matin et le soir pour leur publicité. Il est apparu ainsi une véritable compétition pour les ouvertures entre les différents types d'entreprises juste après l'entrée en vigueur de la nouvelle loi, surtout dans la branche de l'alimentation. La concurrence s'est également développée entre, d'un côté, les magasins spécialisés dans l'ameublement, le matériel électronique, les ordinateurs et l'électronique de divertissement et, de l'autre côté, les grandes surfaces. Bien sûr, la prolongation des heures d'ouverture jusqu'au soir n'a pas vraiment eu d'effet contagieux sur le reste du secteur de service privé et sur le service public. C'est-à-dire qu'il n'est arrivé que très rarement que des succursales de banque ou des institutions publiques comme par exemple des bureaux d'inscription ou des perceptions aient un peu prolongé leurs heures d'ouverture dans des lieux très fréquentés.

Dans l'analyse des effets de la libéralisation des heures d'ouverture, la question de l'évolution du chiffre d'affaires des entreprises du commerce de détail est bien sûr spécialement intéressante. Il est cependant plus facile de poser cette question que d'y répondre. Si des grands magasins ou des commerces spécialisés dans le textile et l'habillement déclarent que, pendant les jours ouvrables normaux, ils réalisent de 15 à $17 \%$ de leur chiffre d'affaires moyen après $18 \mathrm{~h} 30$ et de 25 à $28 \%$ le samedi après $14 \mathrm{~h}$, il s'agit en fait en grande partie d'une comparaison avec les chiffres d'affaires obtenus pendant les heures d'ouverture autorisées avant la nouvelle règlementation. Le résultat de l'étude IFO a montré qu'entre 1995 et 1998 les entreprises qui ouvraient le soir et le samedi après-midi ont déclaré une augmentation du chiffre d'affaires d'environ 3,5\%. En revanche, celles qui sont fermées en général après $18 \mathrm{~h} 30$ ou bien le samedi après 14 h ont dû subir une diminution de $3 \%$ en moyenne.

Ce résultat ne peut être interprété simplement comme résultat de l'ouverture prolongée, car l'évolution du chiffre d'affaires est influencée par un grand nombre de facteurs. Cependant, il n'y a aucun doute sur le fait que les magasins qui ouvrent longtemps peuvent enregistrer une évolution plus avantageuse que les entreprises passives.

Donc, les nouvelles heures d'ouverture n'ont pas seulement eu pour conséquence que les magasins les plus grands appartenant à des chaînes, aient pu améliorer leur position du marché aux frais de moyens ou petits magasins. Les nouvelles heures d'ouverture ont mené aussi à une compétition plus forte entre les grandes surfaces, qui sont prêtes à prolonger leurs heures d'ouverture et qui ont des implantations de type différent. Car désormais, les consommateurs peuvent, sans se presser, comparer les offres faisant l'objet d'une forte promotion, découvrir de nouveaux produits et de nouvelles possibilités de loisir.

24 Cependant, le sujet de la fermeture des magasins reste toujours d'actualité. En juillet 2000, le Städtetag (Union des villes allemandes), soutenu par l'Union fédérale des grandes et moyennes entreprises (BAG), a proclamé qu'il était en faveur de la différenciation des heures d'ouverture entre les magasins des centres ville et ceux qui se situent « auf der grünen Wiese ». Le Städtetag exige que l'on donne la possibilité aux communes de décider d'une prolongation des heures d'ouverture par un règlement valable seulement pour certains secteurs. Ainsi, les heures d'ouverture des magasins 
pourraient être prolongées jusqu'à 22 heures dans les centres-villes et l'attractivité de ces derniers en serait renforcée. D'après le Städtetag, cette prolongation serait nécessaire afin de réagir contre le développement actuel des centres de magasins et de services dans les gares, sans essuyer le reproche d'une évolution non conforme à la Loi fondamentale (Grundgesetz), puisque l'animation des centres-villes est une bonne chose pour tous. En revanche, l'Union principale du commerce de détail allemand (HDE) et l'Union fédérale des entreprises de succursales et des magasins self-service (BFS) prétendent qu'un tel privilège des centres ville serait anticonstitutionnel et exigent la libéralisation totale et en tout lieu des heures d'ouverture pendant les jours ouvrables. Naturellement, il faut attendre pour voir s'il y aura une majorité politique qui se dégagera en faveur de cette dernière proposition ou pour l'initiative du Städtetag.

\section{BIBLIOGRAPHIE}

Pred A. (1977). - The Choreography of Existence - Comments on Hägerstrand's Time-Geography and its Usefulness. Economic Geography 53, 207-221.

Täger U. Chr., Vogler-Ludwig K., Munz S. (1995). - Das deutsche Ladenschlußgesetz auf dem Prüfstand. (= Schriftenreihe des IFO-Instituts für Wirtschaftsforschung Nr. 139) Berlin, München.

Wölk A. (1993). - Städtebauliche Auswirkungen veränderter Ladenschlußzeiten (Dienstleistungsabend). Bonn-Bad Godesberg.

\section{NOTES}

1. Le terme allemand "auf der grünen Wiese» (= sur la prairie verte), sans équivalent en français, veut exprimer : en dehors des agglomérations, loin de tout.

\section{RÉSUMÉS}

Bien que, lors d'un sondage, la majorité des entreprises du commerce de détail ainsi que des consommateurs aient déclaré être opposés à un changement de la loi fixant l'heure de fermeture des magasins (LFFM) de 1956, le législateur a décidé une libéralisation. Cette dernière est entrée en vigueur le 1er novembre 1996 et permet maintenant l'ouverture des magasins jusqu'à 20 heures. Pourtant, les effets sur le plan économique global qui avaient été attendus de cette extension des horaires d'ouverture ne bénéficient pas dans la même mesure à toutes les 
entreprises du commerce de détail. Les avantages diffèrent selon le type d'entreprise ainsi que leur lieu d'implantation.

Although the majority of both retail business operators and consumers indicated opposition to changes in the 1956 law regulating retail operating hours, liberalising legislation was nonetheless enacted which since November 1, 1996, allows for the opening of retail business until 8 p.m. The expected economic effects of this extension of business operations have not been felt by all retailers equally, but are differentiated according to differences in business structures and locations.

Obwohl Einzelhandelsbetriebe wie Verbraucher in Umfragen sich mehrheitlich gegen eine Veränderung des 1956 beschlossenen Ladenschlußgesetzes ausgesprochen hatten, entschloß sich der Gesetzgeber dennoch zu einer Liberalisierung, die seit 1. November 1996 in Kraft getreten ist und nun eine abendliche Ladenöffnung bis 20 Uhr erlaubt. Die von dieser Erwartung der Handlungsspielräume erwarteten gesamtwirtschaftlichen Effekte kommen aber nicht allen Einzelhandelsbetrieben in gleicher Weise zugute, sondern fallen nach Betriebsformen und Standorten unterschiedlich aus.

\section{INDEX}

Mots-clés : commerce de détail, consommateurs, géographie du temps, horaires, RFA

Schlüsselwörter : Bundesrepublik Deutschland, Einzelhandel, Öffnungszeiten, Verbraucher, Zeitgeographie

Keywords : consumers, geography of time, hours, retail business, RFA

\section{AUTEURS}

\section{GÜNTER HEINRITZ}

Geographisches Institut der Technischen Universität, Arcisstr. 21 - 80333 - München 